\title{
Network Analysis of Media Exposure and Psychological Outcomes During the Initial Outbreak of COVID-19 in China
}

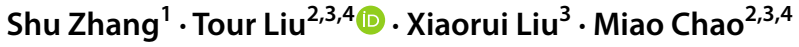

Accepted: 8 December 2021

(C) The Author(s), under exclusive licence to Springer Science+Business Media, LLC, part of Springer Nature 2021

\begin{abstract}
During the initial outbreak of COVID-19 in China, people spent a lot of time viewing or listening to COVID-19-related media content. And according to the conservation of resources theory, COVID-19-related media exposure would be related to multiple psychological outcomes, and different contents would have different associations with different psychological outcomes. Therefore, the present study aimed to clarify these relationships and find out the most important psychological outcome correlated with COVID-19-related media exposure. An online survey was conducted to collect information about individuals' media use behaviors and mental health status. A total of 917 participants were included in further analyses. The results proved that different kinds of content had different relationships with different psychological outcomes. To be more specific, the content about people being heroic and speeches from experts and the authorities were related to increases of positive affect, while reports from hospitals were correlated with increases of death anxiety. Moreover, positive affect was central nodes of the network. The current findings indicated viewing objective and positive content was associated with positive psychological outcomes while viewing negative one was correlated with negative psychological outcomes. Furthermore, positive affect was the most important psychological outcome related to COVID-19-related media exposure.
\end{abstract}

Keywords Media content · COVID-19 · Mental health $\cdot$ Positive affect $\cdot$ Network analysis

Tour Liu

mikebonita@hotmail.com

1 Shanghai Key Laboratory of Mental Health and Psychological Crisis Intervention, School of Psychology and Cognitive Science, East China Normal University, Shanghai 200062, China

2 Key Research Base of Humanities and Social Sciences of the Ministry of Education, Academy of Psychology and Behavior, Tianjin Normal University, Tianjin 300387, China

3 Faculty of Psychology, Tianjin Normal University, Tianjin 300387, China

4 Tianjin Social Science Laboratory of Students' Mental Development and Learning, Tianjin 300387, China 


\section{Introduction}

COVID-19 has spread throughout China since January 2020. By November 7, 2021, over 249 million cases were confirmed globally. To contain the spread of the disease, selfquarantine was suggested by the government on January 20, 2020. Accordingly, media seemed to be the only way where people could get access to COVID-19-related information. According to a national survey of 11,055 people, $99.2 \%$ of the participants were concerned about the pandemic, and up to $45.6 \%$ of them spent more than $3 \mathrm{~h}$ per day viewing COVID-19-related news reports online (Wang et al., 2020). In the meantime, another national survey of over 14,000 people found that depression and anxiety symptoms arose during the initial outbreak of COVID-19 (Guo \& Yang, 2020). These reports indicated that it is necessary to pay attention to people's frequent media use behaviors and mental health during the outbreak.

The relationships between disaster-related media exposure and psychological outcomes had received a lot of attention, and these disasters included natural disasters (e.g., McLeish $\&$ Del Ben, 2008), peacetime terrorist attacks (e.g., Holman et al., 2014), and pandemics (e.g., Mesch et al., 2013). According to the conservation of resources (COR) theory, individuals strive to obtain, retain, foster, and protect resources they value (Hobfoll, 1989; Hobfoll et al., 2018). These resources not only refer to objects they possess (e.g., cars, tools) but also psychological resources (Hobfoll et al., 2018). A previous experiment study found that only exposing to the video clip depicting terror attacks but not experiencing them personally could lead to reductions of individuals' current psychological resources (Zeidner et al., 2011).

The outbreak of COVID-19 had already influenced people's daily life a lot (Bao et al., 2020). Many people were afraid that some terrible things might happen to them, such as losing jobs or getting infected. Even if they did not really get infected or lose their jobs, exposure to COVID-19-related media coverage would engender their feelings of terror (Zeidner et al., 2011), and they would be worried about the fact that they would lose some resources due to the outbreak of COVID-19. The COR theory indicated that the perceived reduction of these resources would, in turn, lead to increases of stress and other negative psychological outcomes (Hobfoll, 1989). Thus, it is convincible that many empirical studies claimed that exposure to disaster-related media coverage was related to increases of stress (Dougall et al., 2005), depression (Ahern et al., 2002), anxiety (Ortiz et al., 2011), and negative affect (Zeidner et al., 2011).

According to the COR theory, the resources people possessed are not isolated from each other. Indeed, there are strong associations among them, and they tend to travel in resource caravans (Hobfoll, 2012). For example, self-esteem, self-efficacy, and optimism are three key personal resources, which are highly intercorrelated (Hobfoll, 2012; Luszczynska et al., 2005). That is, different psychological resources would be elicited or deprived in a similar environment, and they would interact with each other. Accordingly, when watching COVID-19-related media coverage, people would realize the reduction of multiple resources, such as their jobs, optimism, and money. And these resources might not only relate to stress but also to many kinds of psychological outcomes (Zeidner et al., 2011).

Therefore, in addition to the psychological outcomes mentioned above, previous empirical studies also proved that exposure to disaster-related media content could also lead to a pessimistic attitude towards life. In the disaster context, people would always focus on existential concerns such as awareness of their inevitable death (Schrader \& Wann, 1999). And such awareness could lead to increases of death anxiety (Neimeyer et al., 1977) and 
decreases of satisfaction with life (Satici et al., 2020) and meaning in life (Drescher et al., 2012).

However, some recent studies found that disaster-related media content was not only related to negative psychological outcomes (e.g., Chao et al., 2020b). To be more specific, a survey-based study found that certain content, such as images of heroic acts and information about the disaster itself, was correlated with lower odds of PTSD, while other content, such as information about victims, was associated with PTSD (Hall et al., 2019). According to the COR theory, when people are aware of resource loss, they will take action to acquire and conserve resources for survival (Hobfoll et al., 2018). Therefore, it sounds reasonable that people would like to view positive media content (such as heroic acts) to reinforce their confidence in overcoming the pandemic.

Therefore, the first aim of the present study was to explore the associations between different kinds of COVID-19-related media content and multiple psychological outcomes since few previous studies had ever investigated this. And according to the COR theory, we hypothesized that negative COVID-19-related media content would be related to negative psychological outcomes, while positive media content would correlate with positive psychological outcomes.

Furthermore, all the evidence above indicated that exposure to disaster-related media content would correlate with many kinds of psychological outcomes, and these psychological outcomes might also interact with each other. To be more specific, comorbidities might occur when people suffer from one symptom which is closely related to others. However, limited by traditional analysis methods (such as the mediation models and regression analysis), most work done in this domain only focused on a few variables at a time. Therefore, it is necessary to clarify which one or two psychological outcomes are the most important so that people can know exactly about the most important symptom they are experiencing. Besides, it is essential for clinicians to find out the most central psychological factor in this complex relationship network, and pay more attention to these central symptoms to thwart the emergence of comorbidity (Jones et al., 2018).

Therefore, the current study utilized network analysis for two purposes. One purpose was to find out whether negative COVID-19-related media content was related to negative psychological outcomes and positive content correlated with positive psychological outcomes. The other one was to examine the complex relationships among multiple psychological outcomes in an exploratory way and find the central nodes in the correlation network.

\section{Methods}

\section{Procedure and Participants}

From January 20, 2020, self-quarantine was suggested by the government to control the spread of COVID-19, and our study was conducted a week after that. The present survey was internet-based (wj.qq.com). Initially, participants knew about this study through the link shared by several teachers and students from Tianjin Normal University via WeChat Moments (a popular Chinese social media platform). People who saw the link were encouraged to complete the questionnaire and share this link with more people via a snowball sampling method. All the participants had been given consent for using their data in the 
study, and this study was approved by the ethics committee of Tianjin Normal University (code: XL2020-12).

\section{Measures}

Six questionnaires and scales were used to measure media use behaviors and psychological outcomes. We adapted three questionnaires (media use, death anxiety, and meaning in life) to fit the COVID-19 context. To ensure the reliability and validity of the adapted questionnaires, another two groups of participants were investigated. The results of these empirical samples confirmed that these questionnaires were reliable and valid.

\section{Media Use}

COVID-19-related media use questionnaire was composed of six items which were adapted from previous research (Hall et al., 2019). These items assessed the COVID-19-related media content participants viewed, including the severity of the outbreak, knowledge of the disease and prevention, speeches from experts and the authorities, information from acquaintances (via WeChat, Microblog, and so on), reports from hospitals, and people being heroic. One example question is "How often did you view the content about knowledge of the disease and prevention last week?" These six items were rated on a five-point scale of 1 (never) to 5 (always). The sum of these six items represented the media content score. The Cronbach's alpha coefficient in this study was 0.82 .

Confirmatory factor analysis (CFA) was conducted based on the data of another group of 713 participants. The results showed a good fit for one-factor model $\left(\chi^{2}=48.689, d f=8\right.$, $\mathrm{CFI}=0.971, \mathrm{TLI}=0.946, \mathrm{RMSEA}=0.084, \mathrm{SRMR}=0.028)$. And the Cronbach's alpha coefficient was 0.82 .

\section{Positive and Negative Affect}

The Positive Affect and Negative Affect Scale (PANAS) has two subscales. Each subscale is composed of ten words (Watson et al., 1988), such as "interested" for positive affect and "afraid" for negative affect. Participants were asked to what extent they have felt this way during the past week. Items were rated on a five-point scale, from 1 (almost none) to 5 (extremely much). The Chinese version which was also reliable and valid (e.g., X. Chen et al., 2020). With higher scores, people would experience more positive or negative affect. In this study, the Cronbach's alpha coefficients for each subscale were as follows: positive, 0.84 ; and negative, 0.90 .

\section{Satisfaction with Life}

The Satisfaction with Life Scale (SWLS) consisted of five items (Diener et al., 1985). One example item is "The conditions of my life are excellent." Items were rated on a five-point scale, from 1 (totally disagree) to 5 (totally agree). And its Chinese version was confirmed to be reliable and valid (e.g., Xue et al., 2021). With higher scores on this scale, individuals would be more satisfied with their own life. In this study, the Cronbach's alpha coefficient was 0.84 . 


\section{Death Anxiety}

Four items were adapted from the previous death anxiety scale (Templer, 1970) to fit the context of the COVID-19 outbreak. These items were rated on a five-point scale of 1 (totally disagree) to 5 (totally agree). One example question is "I fear dying in this epidemic." Individuals with higher scores indicated they were more concerned about their own safety. In this study, the Cronbach's alpha coefficient was 0.78 .

In the other study of 713 participants, the result of CFA showed a good fit for one-factor model $\left(\chi^{2}=2.765, d f=1, \quad \mathrm{CFI}=0.998, \quad \mathrm{TLI}=0.987, \quad \mathrm{RMSEA}=0.050\right.$, SRMR $=0.009$ ). And the Cronbach's alpha coefficient was 0.79 .

\section{Meaning in Life}

The present meaning in life questionnaire was revised from the previous research (Steger et al., 2006). One example item is "Faced with this epidemic, I still understand my life's meaning." Items were measured on a seven-point scale, from 1 (absolutely untrue) to 7 (absolutely true). Higher scores indicated individuals were more likely to create meaning in his or her own life. In this study, the Cronbach's alpha coefficient was 0.85 .

The results of confirmatory factor analysis (CFA) based on another group of 934 participants showed a good fit for one-factor model $\left(\chi^{2}=10.291, d f=2, \mathrm{CFI}=0.997\right.$, TLI $=0.990$, RMSEA $=0.067$, SRMR =0.009). And the Cronbach's alpha coefficient was 0.91 .

\section{Depression, Anxiety, and Stress}

The Depression Anxiety Stress Scales (DASS-21) has three 7-item subscales for depression, anxiety, and stress (Lovibond \& Lovibond, 1995). Items were measured on a four-point scale, from 1 (never) to 4 (always). Example items include "I was unable to become enthusiastic about anything" for depression; "I experienced trembling (e.g., in the hands)" for anxiety; and "I found it difficult to relax" for stress. The reliability and validity of the Chinese version had been confirmed to be reliable and valid repeatedly (e.g., Chao et al., 2020b). Higher scores indicated higher levels of psychological distress. The Cronbach's alpha coefficients for each subscale in this study were as follows: depression, 0.82; anxiety, 0.78; and stress, 0.83 .

\section{Data Analysis}

\section{Correlation Analysis}

The correlation analysis was conducted by the lavCor argument in lavaan package (version 0.6-9) for $\mathrm{R}$ (version 4.1.1), and the scores of the six kinds of media content were treated as ordered. Polychoric correlations were computed between two ordinal variables, polyserial correlations were computed between one ordinal variable and one continuous variable, and Pearson correlations were computed between two continuous variables. And the corrplot package (version 0.90) was utilized to generate a graphical correlation matrix. 


\section{Correlation Network Analysis}

Correlation networks can describe correlation or partial correlation relationships between variables in quantitative and graphical ways. By means of the graph theory, networks depict the components of a system (i.e., nodes) and the links between these components (i.e., edges). In a correlation network, nodes generally correspond to variables, and edges represent the correlations or partial correlations between variables.

There would be many spurious correlation relationships in the network due to the large number of nodes. To be specific, nodes A and B are not directly related to each other, but they both relate to node C. Accordingly, they can still be statistically significantly correlated. To avoid this misleading result, partial correlation relationships are commonly used to represent the relationships between nodes in a network. Furthermore, it will be difficult to explain this network when there are too many correlations (i.e., edges) in a network. So, Graphical Least Absolute Shrinkage and Selection Operator (GLASSO) model was utilized to shrink small correlation coefficients to zero in the present study. In this way, only strong partial correlations were displayed in the correlation network. The severity of the sparsity parameter $(\gamma)$ was set to 0.5 , attempting a balance between sparseness and sensitivity (J. Chen \& Chen, 2008; Foygel \& Drton, 2010).

As for the quantitative way, there are three most used centrality indices: strength centrality, betweenness centrality, and closeness centrality. Strength centrality is the sum of weights connected to the given node. Betweenness centrality indicates the number of times the given node is on the shortest path between two other nodes. Closeness centrality is the inverse total length of the shortest path from the given node to all other nodes (Opsahl et al., 2010; Zhang et al., 2020, 2021). In the present study, strength centrality measured the total correlation with all variables which directly connected to itself. A node with higher betweenness centrality was more like a "bridge" connecting other variables. Closeness centrality represented how close the focal node was to all other nodes. In general, the higher centrality indices a variable has, the closer it is related to other variables.

The correlation network and the plot depicting centrality indices of each node in the network were both generated by qgraph package (version 1.6.9; Epskamp et al., 2012) in R (version 4.1.1).

\section{Common Method Bias Test}

Harman's single factor test was conducted in IBM SPSS 26.0 to examine the extent of common method bias. Results showed that there were 12 factors with eigenvalues greater than 1 . And the first factor only accounted for $18.49 \%$ of the total variance, suggesting there was no serious common method bias in this study.

\section{Results}

\section{Participant Characteristics and Descriptive Statistics}

Nine hundred and seventeen participants (304 males, 613 females) were included in the present study. And the average age of them was $28.6(S D=9.5$, range $=17-70)$. Among them, 428 participants $(46.7 \%)$ were students, 394 participants $(43.0 \%)$ were employed in 
various industries, 18 participants $(2.0 \%)$ were currently unemployed, and 77 participants (8.4\%) were in other occupations. As for their educational attainment, there were 50 participants $(5.5 \%)$ who had a high school diploma, 84 participants $(9.2 \%)$ who received vocational education, 425 participants $(46.3 \%)$ who received college education, and 358 participants $(39.0 \%)$ who had graduated degree or above.

Descriptive statistics of the variables are summarized in Table 1. Results showed that participants viewed or listened to a lot of COVID-19-related media coverage from January 21,2020 , to January 28 . And they most cared about the severity of the outbreak and knowledge of disease and prevention. Since we did not have a norm to reference and there was no related investigation before the outbreak, we cannot tell whether the participants' psychological status was better or worse.

\section{Correlation Analysis}

Results of correlation analysis (Fig. 1) showed that most of these 14 variables were significantly related to each other. Specifically, the six kinds of media content had significant positive correlations with many psychological outcomes. However, due to the large number of variables, there might be some suspicious correlations. Thus, the following network analysis was conducted based on partial correlation analysis.

\section{Network Analysis}

Figure 2 presents the results of regularized partial correlation network. Visual examination of the network indicated that only death anxiety and positive affect were directly related to media content. It can also be seen from the figure that speeches from experts and the authorities (MC3), depression, and positive affect were directly related to many variables. Furthermore, anxiety, depression, and stress were closely related to each other.

Table 1 Descriptive statistics of the variables $(n=917)$

\begin{tabular}{lcll}
\hline & $M \pm S D$ & Min & Max \\
\hline Media content & $22.30 \pm 3.93$ & 6 & 30 \\
$\quad$ Severity of the outbreak & $3.93 \pm 0.94$ & 1 & 5 \\
Knowledge of disease and prevention & $3.92 \pm 0.82$ & 1 & 5 \\
Speeches from experts and the authori- & $3.70 \pm 0.91$ & 1 & 5 \\
$\quad$ ties & & & \\
Information from acquaintances & $3.65 \pm 0.96$ & 1 & 5 \\
$\quad$ Reports from hospitals & $3.54 \pm 0.91$ & 1 & 5 \\
$\quad$ People being heroic & $3.56 \pm 0.93$ & 1 & 5 \\
Positive affect & $28.59 \pm 6.02$ & 10 & 50 \\
Negative affect & $22.91 \pm 7.42$ & 10 & 50 \\
Satisfaction with life & $12.10 \pm 3.77$ & 5 & 25 \\
Death anxiety & $14.38 \pm 3.83$ & 4 & 20 \\
Meaning in life & $21.43 \pm 4.39$ & 4 & 28 \\
Depression & $10.77 \pm 3.27$ & 7 & 26 \\
Anxiety & $11.07 \pm 3.02$ & 7 & 26 \\
Stress & $12.32 \pm 3.45$ & 7 & 26 \\
\hline
\end{tabular}


1. Severity of the outbreak

2. Knowledge of disease and prevention

3. Speeches from experts and the authorities

4. Information from acquaintances

5. Reports from hospitals

6. People being heroic

7. Satisfaction with life

8. Death anxiety

9. Meaning in life

10. Positive affect

11. Negative affect

12. Depression

13. Anxiety

14. Stress

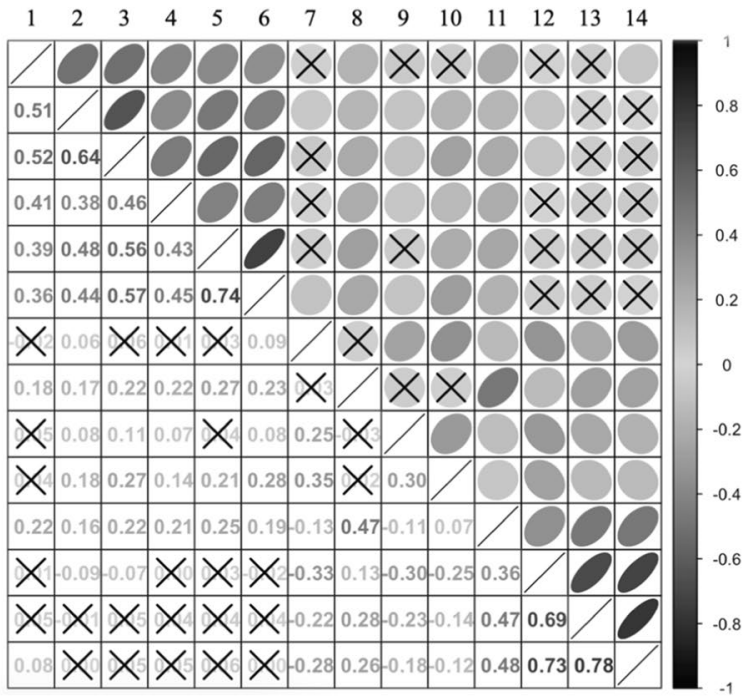

Fig. 1 Graphical correlation matrix. The correlation coefficients with " $x$ " indicate that $p$ value is higher than .05. The darker the color of the figure and the number are, the stronger the correlation is

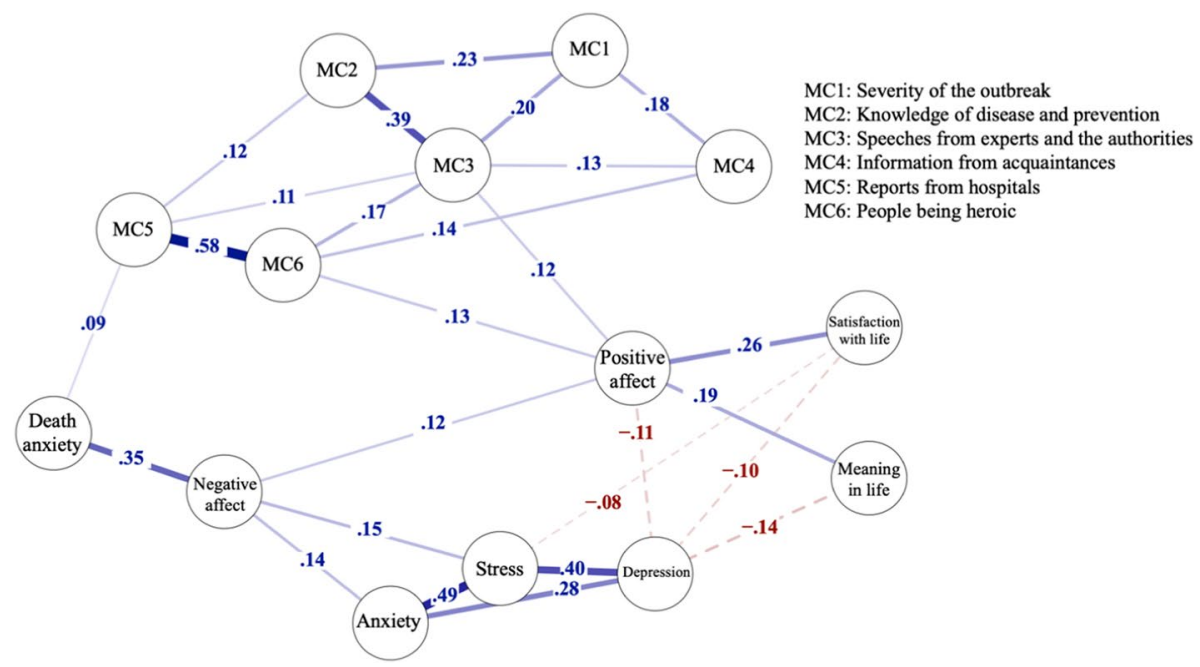

Fig. 2 Correlation network of 14 variables. Nodes represent variables. Solid blue edges represent positive correlations between variables, and dotted red edges represent negative correlations. The thicker the edge is, the stronger the correlation is. See the digital version of the paper for the color version of this figure

Figure 3 displays Z-scores of the node centrality indices. The results illustrated that positive affect had the highest betweenness centrality and closeness centrality, which meant it was a central node. Stress and MC3 had the highest strength centrality. Besides, depression also had higher betweenness centrality and strength centrality, which indicated it was also an important node in the network, but not as important as positive affect. Moreover, death 


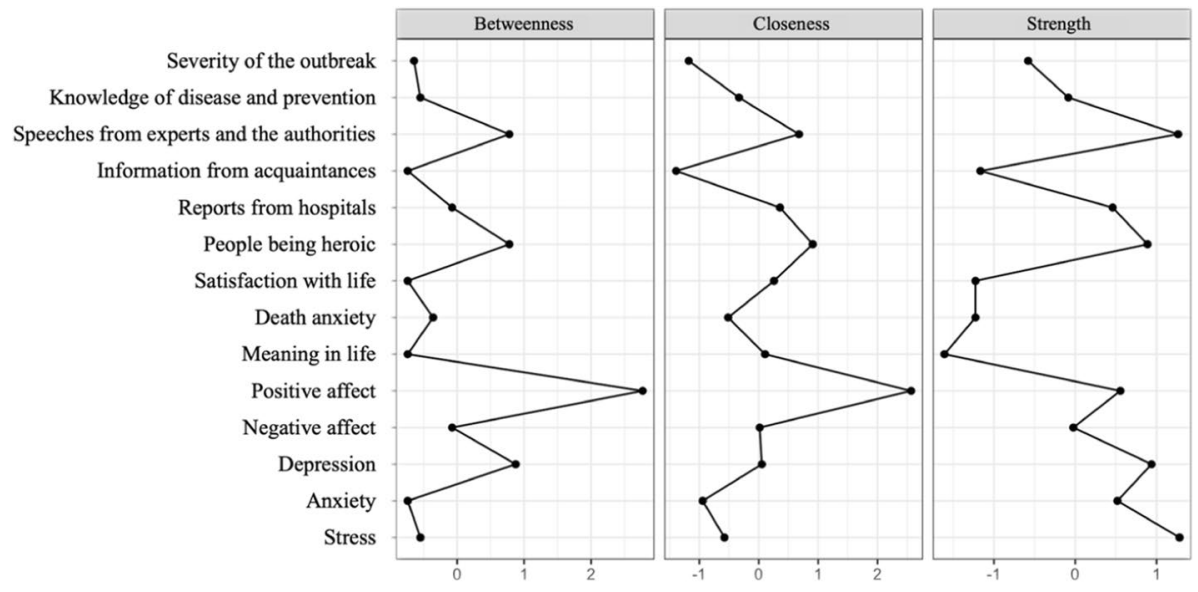

Fig. $3 Z$-scores of node centrality indices

anxiety, meaning in life, and satisfaction with life had lower centrality, distancing from other variables.

\section{Discussion}

The present study had two aims: one was to explore the relationships between exposure to different kinds of COVID-19-related media content and multiple psychological outcomes; the other one was to find the central psychological outcomes related to COVID-19-related media exposure. The results of this study showed that exposure to the content about speeches from experts and the authorities and people being heroic was positively correlated with positive affect, while the content about reports from hospitals was positively correlated with death anxiety. Moreover, network structures demonstrated that positive affect was the central node of this correlation network, and depression was less important than positive affect.

In line with our hypothesis, different media content was found to have different associations with psychological outcomes. To be more specific, during the initial outbreak of COVID-19, people knew little things about this virus, and hospitals could not take good care of all the patients due to the lack of medical staff and medical supplies (Bao et al., 2020). So, according to the COR theory and terror management theory (TMT), reports from hospitals would make people worried about their safety (Greenberg et al., 1997). As the previous study pointed out, awareness of one's own mortality increased by exposure to disaster-related media coverage would result in feelings of helplessness and a pessimistic attitude towards life (Zeidner et al., 2011). Therefore, viewing the content about reports from hospitals was associated with the increase of death anxiety.

The present results also illustrated that the content about people being heroic and speeches from experts and the authorities had positive correlations with positive affect. This supported the previous study which found viewing more information about Typhoon Hato itself and images of heroic acts was a protective factor for PTSD (Hall et al., 2019). During this anti-COVID-19 campaign, viewing heroic acts and learning knowledge about COVID-19 would increase people's sense of control and made them have full confidence 
in winning this "war" (Lazarus \& Folkman, 1984). And this confidence made up for the reduction of resources, which increases positive affect.

Not like most previous studies, positive and negative affect were positively correlated in the present study. Indeed, they were proved to be two independent processes (Grzybowski et al., 2014; Watson et al., 1988). That meant they do not have to be negatively related all the time. In the present study, the positive association between positive and negative affect might be mediated by the content about reports from hospitals, the content about people being heroic, and death anxiety according to the correlation network.

Moreover, inconsistent with other studies, media content had no direct correlations with depression (e.g., Busso et al., 2014; Pfefferbaum et al., 2014), anxiety (e.g., Ortiz et al., 2011), stress (e.g., Dougall et al., 2005), satisfaction with life (e.g., Satici et al., 2020), and meaning in life (e.g., Zeidner et al., 2011) in the correlation network. This discrepancy might be explained by the analysis methods used. Take the relationship between stress and the content about the severity of the outbreak as an example. Based on the results of polyserial correlation analysis, they were positively correlated with each other. However, when controlling other variables, no direct relationship existed. Therefore, the relationship between the content about the severity of the outbreak and stress might be mediated by other variables. These mediation effects can be discussed further in future studies.

Consistent with previous research, positive affect was directly correlated with increased satisfaction with life (Hurley \& Kwon, 2013) and meaning in life (Chao et al., 2020a, b; Hicks et al., 2012), and decreased depression (Headey et al., 1993), suggesting that positive affect did have significant associations with many other psychological outcomes in multiple contexts. Besides, positive affect had the highest betweenness and closeness centrality, indicating it acted as a bridge to the connections of other variables and was also close to other nodes. That meant positive affect was the psychological outcome most relevant to COVID-19-related media exposure. Moreover, due to its central position in the network, it acted as a bridge connecting media content with other psychological outcomes. Therefore, clinicians can suggest people view more positive or objective media content (such as heroic acts and knowledge about the disease) to improve resilience and avoid suffering psychological distress during the outbreak.

Moreover, depression had the second-highest betweenness centrality and higher strength centrality, having five direct relationships with other nodes. These results reminded people that it was also an important psychological outcome, especially a negative one, although it had no direct relationship with all the six kinds of media content. Therefore, when suffering from depression, people should not view more negative media coverage; lest depression would lead to more other negative psychological outcomes. Besides, although stress had the highest strength centrality, it was not the central node in the network with low betweenness and closeness centrality. To be more specific, the nodes connected with it were not central nodes, nor was it on the shortest path connecting any other two nodes. The highest strength centrality of stress was contributed by the strong correlations with depression and anxiety, while depression and anxiety were not central nodes either. Therefore, stress was not a central node despite having the highest strength centrality.

\section{Implications and Limitations}

In conclusion, the present study clarified the different associations between different kinds of COVID-19-related media content and multiple psychological outcomes. Besides, the 
results also found out the most important psychological outcomes related to media exposure by using network analysis.

The present results proved that positive and objective COVID-19-related media content was correlated with positive psychological outcomes, while some negative content was related to negative psychological outcomes. Moreover, network structures informed potential comorbidities and demonstrated positive affect were the most important role in the network. Therefore, to avoid increases in negative psychological outcomes, people should not pay more attention to the negative content. For those who are pessimistic about this pandemic, clinicians can advise them to view more objective and positive media content, which might improve their resilience.

There are also several limitations of this study. First, directions of effects cannot be established in the undirected network. However, the relationships between media use behaviors and psychological outcomes might be bidirectional (Aalbers et al., 2019). Therefore, further research should collect time-series data and use multilevel vector autoregressive time-series models to warrant potential causal effects. Secondly, individuals' media use behaviors and mental health might be affected by the number of confirmed cases of the city where they lived. But limited by the small sample size, we did not take this into consideration in the present study. Finally, self-report measures may be inaccurate. Previous research found that sometimes individuals might overestimate the time they spent on social media (Junco, 2013). Thus, further studies can use self-tracking applications or software to assess the accurate time that participants spent on different types of media.

Funding This work was supported by Tianjin Philosophy and Social Science Project (TJJXQN20-003).

\section{Declarations}

Ethics Approval Approval was obtained from the ethics committee of Tianjin Normal University (code: XL2020-12). The procedures used in this study adhere to the tenets of the Declaration of Helsinki.

Consent to Participate All the participants had given consent for their data to be used in the research.

Conflict of Interest The authors declare no competing interests.

\section{References}

Aalbers, G., McNally, R. J., Heeren, A., de Wit, S., \& Fried, E. I. (2019). Social media and depression symptoms: A network perspective. Journal of Experimental Psychology: General, 148(8), 1454-1462.

Ahern, J., Galea, S., Resnick, H., Kilpatrick, D., Bucuvalas, M., Gold, J., \& Vlahov, D. (2002). Television images and psychological symptoms after the September 11 terrorist attacks. Psychiatry, 65(4), 289-300.

Bao, Y., Sun, Y., Meng, S., Shi, J., \& Lu, L. (2020). 2019-nCoV epidemic: Address mental health care to empower society. The Lancet, 395(10224), e37-e38.

Busso, D. S., McLaughlin, K. A., \& Sheridan, M. A. (2014). Media exposure and sympathetic nervous system reactivity predict PTSD symptoms after the Boston Marathon bombings. Depression and Anxiety, 31(7), 551-558.

Chao, M., Chen, X., Liu, T., Yang, H., \& Hall, B. J. (2020a). Psychological distress and state boredom during the COVID-19 outbreak in China: The role of meaning in life and media use. European Journal of Psychotraumatology, 11(1), 1769379.

Chao, M., Xue, D., Liu, T., Yang, H., \& Hall, B. J. (2020b). Media use and acute psychological outcomes during COVID-19 outbreak in China. Journal of Anxiety Disorders, 74, 102248. 
Chen, J., \& Chen, Z. (2008). Extended Bayesian information criteria for model selection with large model spaces. Biometrika, 95(3), 759-771.

Chen, X., Liu, T., Li, P., Wei, W., \& Chao, M. (2020). The relationship between media involvement and death anxiety of self-quarantined people in the COVID-19 outbreak in China: The mediating roles of empathy and sympathy. OMEGA - Journal of Death and Dying. https://doi.org/10.1177/00302 22820960283

Dougall, A. L., Hayward, M. C., \& Baum, A. (2005). Media exposure to bioterrorism: Stress and the anthrax attacks. Psychiatry, 68(1), 28-42.

Drescher, C. F., Baczwaski, B. J., Walters, A. B., Aiena, B. J., Schulenberg, S. E., \& Johnson, L. R. (2012). Coping with an ecological disaster: The role of perceived meaning in life and self-efficacy following the gulf oil spill. Ecopsychology, 4(1), 56-63.

Epskamp, S., Cramer, A. O. J., Waldorp, L. J., Schmittmann, V. D., \& Borsboom, D. (2012). qgraph: Network visualizations of relationships in psychometric data. Journal of Statistical Software, 48(4), $1-18$.

Foygel, R., \& Drton, M. (2010). Extended Bayesian information criteria for Gaussian graphical models. In J. D. Lafferty, C. K. I. Williams, J. Shawe-Taylor, R. S. Zemel, \& A. Culotta (Eds.), Advances in Neural Information Processing Systems 23 (NIPS 2010) (pp. 2020-2028).

Greenberg, J., Solomon, S., \& Pyszczynski, T. (1997). Terror management theory of self-esteem and cultural worldviews: Empirical assessments and conceptual refinements. In M. P. Zanna (Ed.), Advances in Experimental Social Psychology (Vol. 29, pp. 61-139). Academic Press.

Grzybowski, S. J., Wyczesany, M., \& Kaiser, J. (2014). The influence of context on the processing of emotional and neutral adjectives - An ERP study. Biological Psychology, 99, 137-149.

Guo, L., \& Yang, F. (2020). An investigation report on public psychological status during the outbreak of COVID-19. Retrieved Mar 29, 2020, from https://mp.weixin.qq.com/s/lXEDuWaIGK6tCJdIcItjCQ

Hall, B. J., Xiong, Y. X., Yip, P. S. Y., Lao, C. K., Shi, W., Sou, E. K. L., Chang, K., Wang, L., \& Lam, A. I. F. (2019). The association between disaster exposure and media use on post-traumatic stress disorder following Typhoon Hato in Macao. China. European Journal of Psychotraumatology, 10(1), 1558709.

Headey, B., Kelley, J., \& Wearing, A. (1993). Dimensions of mental health: Life satisfaction, positive affect, anxiety and depression. Social Indicators Research, 23, 63-82.

Hicks, J. A., Trent, J., Davis, W. E., \& King, L. A. (2012). Positive affect, meaning in life, and future time perspective: An application of socioemotional selectivity theory. Psychology and Aging, 27(1), 181-189.

Hobfoll, S. E. (1989). Conservation of resources: A new attempt at conceptualizing stress. American Psychologist, 44(3), 513-524.

Hobfoll, S. E. (2012). Conservation of resources and disaster in cultural context: The caravans and passageways for resources. Psychiatry: Interpersonal and Biological Processes, 75(3), 227-232.

Hobfoll, S. E., Halbesleben, J., Neveu, J. P., \& Westman, M. (2018). Conservation of resources in the organizational context: The reality of resources and their consequences. Annual Review of Organizational Psychology and Organizational Behavior, 5, 103-128.

Holman, E. A., Garfin, D. R., \& Silver, R. C. (2014). Media's role in broadcasting acute stress following the Boston Marathon bombings. Proceedings of the National Academy of Sciences of United States of America, 111(1), 93-98.

Hurley, D. B., \& Kwon, P. (2013). Savoring helps most when you have little: Interaction between savoring the moment and uplifts on positive affect and satisfaction with life. Journal of Happiness Studies, $14,1261-1271$.

Jones, P. J., Mair, P., Riemann, B. C., Mugno, B. L., \& McNally, R. J. (2018). A network perspective on comorbid depression in adolescents with obsessive-compulsive disorder. Journal of Anxiety Disorders, 53, 1-8.

Junco, R. (2013). Comparing actual and self-reported measures of Facebook use. Computers in Human Behavior, 29(3), 626-631.

Lazarus, R. S., \& Folkman, S. (1984). Stress appraisal and coping. Springer.

Lovibond, P. F., \& Lovibond, S. H. (1995). The structure of negative emotional states: Comparison of the Depression Anxiety Stress Scales (DASS) with the Beck Depression and Anxiety Inventories. Behaviour Research and Therapy, 33(3), 335-343.

Luszczynska, A., Gutiérrez-Doña, B., \& Schwarzer, R. (2005). General self-efficacy in various domains of human functioning: Evidence from five countries. International Journal of Psychology, 40(2), 80-89.

McLeish, A. C., \& Del Ben, K. S. (2008). Symptoms of depression and posttraumatic stress disorder in an outpatient population before and after hurricane Katrina. Depression and Anxiety, 25(5), 416-421. 
Mesch, G. S., Schwirian, K. P., \& Kolobov, T. (2013). Attention to the media and worry over becoming infected: The case of the Swine Flu (H1N1) Epidemic of 2009. Sociology of Health and Illness, 35(2), 325-331.

Neimeyer, R. A., Dingemans, P. M. A. J., \& Epting, F. R. (1977). Convergent validity, situational stability and meaningfulness of the threat index. Omega, 8(3), 251-265.

Opsahl, T., Agneessens, F., \& Skvoretz, J. (2010). Node centrality in weighted networks: Generalizing degree and shortest paths. Social Networks, 32(3), 245-251.

Ortiz, C. D., Silverman, W. K., Jaccard, J., \& La Greca, A. M. (2011). Children's state anxiety in reaction to disaster media cues: A preliminary test of a multivariate model. Psychological Trauma: Theory, Research, Practice, and Policy, 3(2), 157-164.

Pfefferbaum, B., Newman, E., Nelson, S. D., Nitiéma, P., Pfefferbaum, R. L., \& Rahman, A. (2014). Disaster media coverage and psychological outcomes: Descriptive findings in the extant research. Current Psychiatry Reports, 16(9), 464.

Satici, B., Gocet-Tekin, E., Deniz, M. E., \& Satici, S. A. (2020). Adaptation of the Fear of COVID-19 Scale: Its association with psychological distress and life satisfaction in Turkey. International Journal of Mental Health and Addiction.

Schrader, M., \& Wann, D. (1999). High-risk recreation: The relationship between participant characteristics and degree of involvement. Journal of Sport Behavior, 22(3), 426-441.

Steger, M. F., Frazier, P., Kaler, M., \& Oishi, S. (2006). The meaning in life questionnaire: Assessing the presence of and search for meaning in life. Journal of Counseling Psychology, 53(1), 80-93.

Templer, D. I. (1970). The construction and validation of a Death Anxiety Scale. The Journal of General Psychology, 82, 165-177.

Wang, J., Gao, W., Chen, M., Ying, X., Tan, X., \& Liu, X. (2020). A survey on psychological status of the public during the COVID-19 outbreak in China. Retrieved Mar 29, 2020, from https://mp.weixin.qq. com/s/KH7Hfx-NnJQcJwWw_jc_IA

Watson, D., Clark, L. A., \& Tellegen, A. (1988). Development and validation of brief measures of positive and negative affect: The PANAS scales. Journal of Personality and Social Psychology, 54(6), 1063-1070.

Xue, D., Liu, T., Chen, X., Liu, X., \& Chao, M. (2021). Data on media use and mental health during the outbreak of COVID-19 in China. Data in Brief, 35, 106765.

Zeidner, M., Ben-Zur, H., \& Reshef-Weil, S. (2011). Vicarious life threat: An experimental test of Conservation of Resources (COR) theory. Personality and Individual Differences, 50(5), 641-645.

Zhang, S., Li, Y., Ren, S., \& Liu, T. (2021). Associations between undergraduates' interpersonal relationships and mental health in perspective of social network analysis. Current Psychology. https://doi.org/ 10.1007/s12144-021-01629-3

Zhang, S., Liu, T., Xia, F., \& Li, Y. (2020). A social network analysis approach to interpersonal relationship and mental health in college students. Chinese Mental Health Journal, 34(10), 855-859.

Publisher's Note Springer Nature remains neutral with regard to jurisdictional claims in published maps and institutional affiliations. 